\title{
Anatomical and Electrophysiological Validation of an Atlas for Neurosurgical Planning
}

\author{
M. Mallar Chakravarty ${ }^{1}$, Abbas F. Sadikot ${ }^{1,2}$, Jurgen Germann ${ }^{1}$, \\ Gilles Bertrand $^{1,2}$, and D. Louis Collins ${ }^{1}$ \\ ${ }^{1}$ McConnell Brain Imaging Centre \\ ${ }^{2}$ Division of Neurosurgery, Montreal Neurological Institute, \\ 3801 University Street, Montreal, Quebec, Canada, H3A 2B4
}

\begin{abstract}
Digital brain atlases can be used in conjunction with magnetic resonance imaging (MRI) and computed tomography (CT) for planning and guidance during neurosurgery. Digital atlases are advantageous, since they can be warped nonlinearly to fit each patient's unique anatomy.

Two atlas-to-patient warping techniques are compared in this paper. The first technique uses an MRI template as an intermediary to estimate a nonlinear atlas-to-patient transformation. The second, is novel, and uses a pseudo-MRI volume, derived from the voxel-label-atlas, to estimate the atlas-to-patient transformation directly. Manual segmentations and functional data are used to validate the two methods.
\end{abstract}

\section{Introduction}

Print atlases were the first visualization tools used to identify surgical targets for functional neurosurgery [16 18. In order to better visualize patient anatomy, digital atlases derived from print atlases are used in conjunction with imaging data to further enhance the visualization of targets in the subcortical nuclei 2 217 15 10 5]. Despite recent advances in medical imaging which allow improved visualization of the thalamus [714], most clinical MRI volumes lack the contrast and resolution required for proper visualization of all subcortical nuclei.

Groups who have developed atlas-to-patient warping techniques have used linear transformations in order to match the atlas to pre-operative patient MRI volumes [2 15]. Recently, nonlinear atlas warping has been used to account for local differences between the atlas and patient anatomy [17.5], and used for neurosurgical planning, to analyze intra-operative electrophysiological data in the thalamus and the internal capsule 89, and the analysis of thalamic lesions [1]. This paper analayzes the two different nonlinear atlas-to-subject warping techniques to determine which is most accurate for surgical planning.

In this paper, we use a new high resolution atlas that contains multiple registered representions of the 105 atlas structures [45]. The atlas is derived from a single set of segmented serial histological data taken from a single subject. The segmentation contours are used to derive a voxel-label-atlas, whose intensities are modified to create a pseudo-MRI. Figure 1 shows the histological volume, 

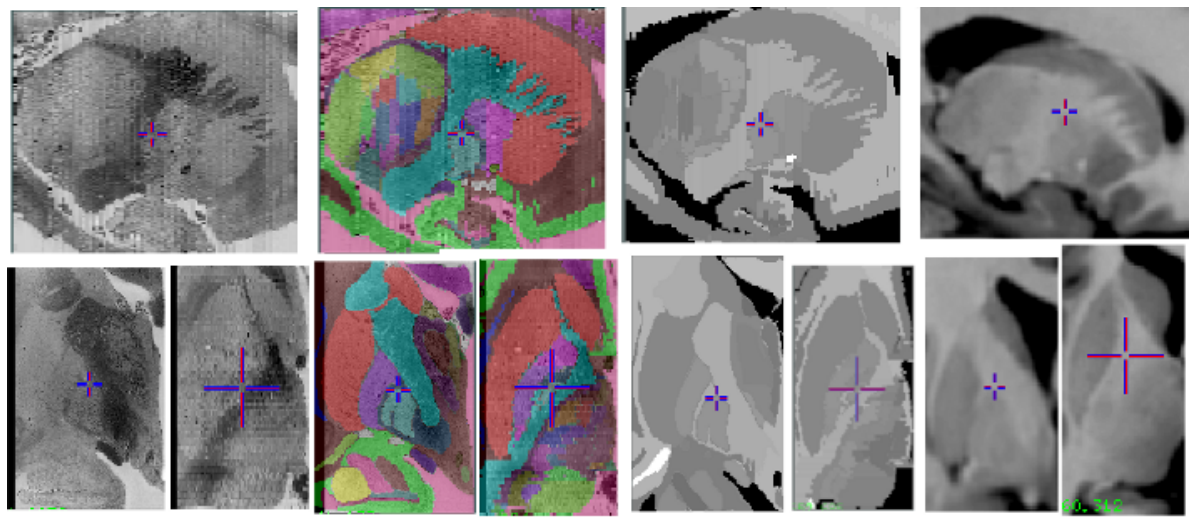

Fig. 1. From Left to Right: Reconstructed histological volume, histological volume with voxel-label-atlas overlaid, pseudo-MRI, and the Colin27 MRI average.
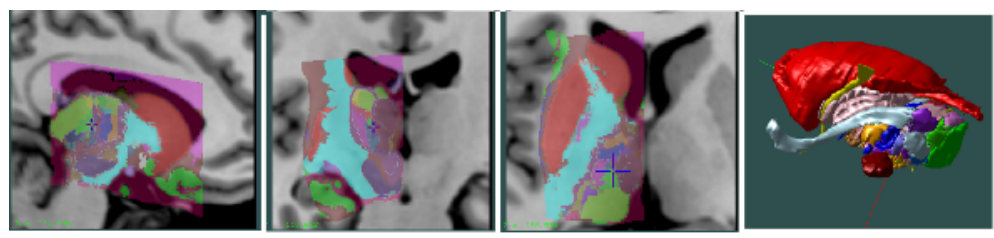

Fig. 2. First three panels: Voxel-label atlas warped to fit the Colin27 MRI average. Right: 3D geometric atlas.

the histological volume with the voxel-label-atlas overlaid, and the pseudo-MRI. Figure 2 shows the voxel-label-atlas warped onto the Colin27 MRI average template [13] and the 3D geometric atlas [5].

This paper evaluates the accuracy of two different atlas-to-patient nonlinear warping techniques presented in Section 2, Section 3 discusses the anatomical and electrophysiological validation criteria. The results of this evaluation are given in Section 4

\section{Atlas-to-Patient Warping Techniques}

Two atlas-to-patient warping techniques have been developed to warp the atlas described in [5] to patient data. Both techniques begin by estimating a 9 parameter linear transformation (consisting of 3 translations, 3 rotations, and 3 scales) to transform the atlas into the native space of the MRI data. This transformation is used as the input for the nonlinear transformation estimation.

Nonlinear warping is used in order to account for any local differences between the anatomy of the atlas and patient data. Both techniques use the ANIMAL algorithm to estimate the nonlinear transformation used to warp the atlas to fit patient data (see section 2.1). 
In the first technique, described in Section 2.2, the Colin27 MRI average is used as an intermediate template in order to estimate a nonlinear transformation. In the original work of St-Jean [17, a thin plate spline was used to align the digital atlas data to the Colin27 template [13. To address limitations of the previous landmark-based technique, the ANIMAL intensitybased image registration technique was used to align the the new atlas with the Colin27 template. Since similar intensities between the atlas and template images were required, a pseudo-MRI was created from the voxel-label-atlas by mapping each label to an intensity from the analogous structure found in the Colin27 MRI average template volume (see Fig. (1). This procedure is validated in section 3.1.

The use of an atlas-based pseudo-MRI inspired the second atlas-to-patient customization technique, presented in Section 2.3. This technique estimates a nonlinear atlas-to-patient transformation directly, without using any intermediate steps.

\subsection{The ANIMAL Registration Algorithm}

ANIMAL is an iterative algorithm [6] which estimates a 3D deformation field which matches a source volume to a target volume. The algorithm is divided into two steps. The first step is the outer loop, where large deformations are estimated on data which has been blurred using a Gaussian kernel with of large full-width-at-half-maximum (FWHM). These larger deformations are then input to subsequent steps where the fit is refined by estimating smaller deformations on data blurred with a smaller FWHM.

Each step in the outer loop contains an inner-loop where the the nonlinear transformation which maximizes the similarity between a source and a target volume is estimated. The inner loop consists of two steps: calculating a deformation at each node which will maximize the local similarity measure, and the second is a smoothing step to ensure that a continuous deformation field has been estimated. For further details on ANIMAL's parameters, the reader is referred to [5]6]. For each step in the warping techniques presented in Sections 2.2 and 2.3 the same weight, stiffness, and similarity values are used $(1,1$, and 0.3 respectively), and use the cross-correlation objective function.

\subsection{MRI Template Based Warping}

As mentioned in Section 1 the atlas has been nonlinearly warped to the Colin27 MRI average [13, which now serves as a template for atlas-to-patient registration. Here, ANIMAL is used to compute the nonlinear transformation required to align the Colin27 template MRI volume with the patient's MRI. The standard parameters used for the registration strategy are shown in Table 1. Once the transformation is estimated, it is applied to the atlas to customize it onto the patient's anatomy. This can be seen in the top path of the flowchart of Fig. 3. 
Table 1. ANIMAL parameters used for template based atlas-to-subject nonlinear transformation estimation

\begin{tabular}{|c||c|c|c|c|}
\hline FWHM $(\mathrm{mm})$ & Step Size $(\mathrm{mm})$ & Sub Lattice Diameter & Sub Lattice & Iterations \\
\hline \hline 8 & 8 & 24 & 6 & 30 \\
\hline 8 & 4 & 12 & 4 & 30 \\
\hline 4 & 2 & 6 & 6 & 10 \\
\hline
\end{tabular}

\subsection{Pseudo-MRI Based Warping}

In the novel customization procedure, the atlas pseudo-MRI is used as the source image in order to estimate the atlas-to-patient nonlinear transformation. Since the pseudo-MRI is derived directly from the anatomy of the atlas, any errors incurred by warping the atlas to an intermediate template are now eliminated. During the nonlinear transformation step, small deformations are estimated to warp the atlas to the target patient MRI volume. No blurring is required due to the homogeneous intensities for each structure represented in the pseudo-MRI. A hierarchical registration strategy is still used. The nonlinear transformation estimation procedure can be seen in the bottom path of the flowchart in Fig. 3 . The registration parameters are shown in Table 2.

\section{Template based warping}

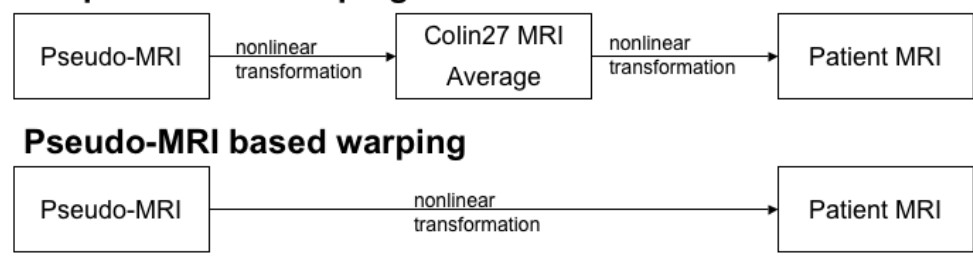

Fig. 3. Flow chart for atlas-to-patient nonlinear transformation estimation. The top path shows the template based atlas-to-patient warping technique. The atlas is registered to the Colin27 MRI template using the pseudo-MRI. The template-to-patient transformation is estimated and then applied to the atlas. The bottom path, demonstrates how the pseudo-MRI based technique bypasses the need to register the atlas to a patient MRI volume by directly estimating an atlas-to-patient transformation.

Table 2. ANIMAL parameters used for template based atlas-to-subject nonlinear transformation estimation

\begin{tabular}{|c||c|c|c|c|}
\hline Step & Step Size (mm) & Sub Lattice Diameter & Sub Lattice & Iterations \\
\hline \hline 1 & 4 & 8 & 6 & 10 \\
\hline 2 & 2 & 6 & 6 & 10 \\
\hline 3 & 1 & 6 & 3 & 10 \\
\hline
\end{tabular}




\section{Atlas-to-Patient Warping Evaluation}

The atlas to patient warping techniques presented in Sections 2.2 and 2.3 were evaluated using clinical T1-weighted pre-operative image data from 10 surgical candidates: 5 subthalamic stimulation cases (5 males) and 5 thalamotomy (3 males and 2 females, 3 left and 2 right thalamotomy) cases with stereotactic headframe attached. All MRI volumes were acquired between 1997 and 2002 . The following sections present anatomical and functional validation for each estimated atlas-to-patient transform.

\subsection{Anatomical Validation}

Since a gold standard for anatomical validation was not available, manual structure segmentations were used. To estimate inter-rater variability, three expert raters identified the striatum, the thalamus, and the globus pallidus in the MRI data. For each structure in each MRI volume, the consensus label (ie: all voxels labelled by at least two raters for each structure) was considered as "the ground truth". This consensus label was verified against the analogous atlas structure by determining the Kappa score $(\kappa)$ :

$$
\kappa=\frac{2 a}{(2 a+b+c)},
$$

where $a$ is the number of voxels common to the rater and the warped atlas, and $b+c$ represents the sum of the voxels uniquely identified by either the rater or the atlas warping technique.

The Kappa score is extremely sensitive to changes in two different labels. Figure 4 shows the striatum from the atlas shown in Figs 1 and 2, If the striatum is translated by $0.5 \mathrm{~mm}$ in all three dimensions, the Kappa score decreases from 1 (for the label on itself) to 0.87 . If the striatum if translated by $1 \mathrm{~mm}$ in all three dimension, then the Kappa score decreases further to 0.81. Typically, scores
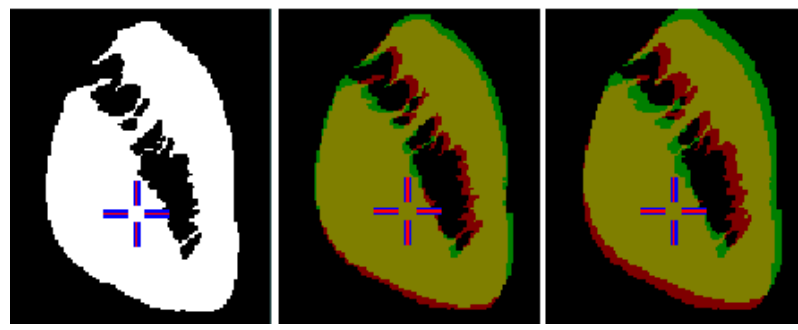

Fig. 4. Changing Kappa values for a displaced Striatum. From left to right Coronal slice through the original striatum defined by the atlas, the striatum translated by $0.5 \mathrm{~mm}$ in all three dimension $(\kappa=0.87)$, and striatum displaced by $1 \mathrm{~mm}$ in all three dimensions $(\kappa=0.81)$. In the middle and right most images, the label in red represents the original striatum, and the label in green represents the translated striatum, and yellow, the overlap between the two. 
greater than 0.7 are deemed acceptable in the segmentation and classification literature.

Two validations were completed. The first quantifies the pseudo-MRI based customization of the atlas data on the Colin27 target, and thus establishes an upper bound for atlas-to-patient warping quality when using the Colin27 MRI template-based warping method. The second anatomical validation was done using the pre-operative data from the 5 subthalamic stimulation cases.

\subsection{Functional Validation}

The functional validation uses intra-operative recordings from the 5 patients who underwent thalamotomies to relieve symptoms of Parkinsons disease. In order to determine the lesion location, surgeons typically locate the sensory thalamus using electrical stimulation. By assessing the patients response to different levels of electrical stimulation $(1 \mathrm{~V}, 0.75 \mathrm{~V}, 0.5 \mathrm{~V}, 0.25 \mathrm{~V})$, the location of the sensory thalamus can be established within the coordinate system established by the stereotactic frame mounted on the patient's head. Using the surgical guidance software [17, the lesion target location, the extension of the electrical stimulator, angles of declination and azimuth, and the probe location frame-space coordinate, the framespace coordinates of the tip of stimulator can be found. For both atlas-to-subject warping techniques, the locations of stimulation point were warped onto the Colin27 MRI average or the pseudo-MRI using the inverse atlas-to-subject transformation. Points from patients who underwent right thalamotomies were reflected to the left side of brain. Only stimulations eliciting a sensory response were used for this validation, yielding a total of 36 functional data points. The warped functional data points were analyzed by determining how many fell inside the 3D boundary defined by the sensory thalamus, and by comparing the distance between the centroid of the warped data points and the center of gravity of the volume defined by the sensory thalamus.

\section{Results}

The first anatomical validation compares the atlas label to manual rater labels of the the Colin27 MRI template to validate the initial atlas-to-template warp. To establish a baseline, each of the raters labels is compared to the consensus label and shown in the first row of Table 3. Kappa scores show excellent agreement between raters for the striatum and thalamus, however the globus pallidus appears to be more difficult to manually segment as demonstrated by the lower kappa value, probably due to difficulty in identification of the posterior border that has lower contrast in the MRI image. Comparisons between the consensus label and the warped atlas, also show excellent agreement for the striatum and the thalamus, but as in the case for the manual raters, it is difficult to reach a consensus on the location of the globus pallidus. These scores indicate how well raters agree with the atlas structures defined on the Colin27 MRI template and serve as the upper limit in the kappa value when comparing the patientcustomized atlas labels below. 
Table 3. Top: Kappa scores to assess the overlap between initial warp to the Colin27 template and the rater-defined consensus label. The mean kappa is comparing the rater label overlap to the consensus label is also given. Bottom: The mean kappa comparing the rater label overlap to the mean label is also given.

\begin{tabular}{|c||c|c|c|}
\hline Structures & Striatum & Thalamus & Globus Pallidus \\
\hline \hline Rater-to-Template $(\bar{\kappa})$ & 0.95 & $0.95(.93-.96)$ & $0.82(.76-.96)$ \\
\hline Pseudo-to-Template $(\kappa)$ & 0.88 & 0.82 & 0.69 \\
\hline
\end{tabular}

\begin{tabular}{|c||c|c|c|}
\hline Structure & Striatum & Thalamus & Globus Pallidus \\
\hline \hline Rater-to-Consensus $(\bar{\kappa})$ & 0.88 & 0.88 & 0.69 \\
\hline Pseudo-to-Consensus $(\bar{\kappa})$ & 0.79 & 0.74 & 0.68 \\
\hline Template-to-Consensus $(\bar{\kappa})$ & 0.80 & 0.71 & 0.63 \\
\hline
\end{tabular}

The second validation compares the template-based and the pseudo-MRI based warping procedure against the manual rater labels on each of the 5 subthalamic stimulation cases. All comparison are done against the baseline established by consensus labels. Kappa values for these comparisons are shown in the top row of the second table in Table 3 . Once again kappa values show excellent agreement between raters, except for in the globus pallidus. Comparisons between the results of the pseudo-MRI based warping technique and templatebased warping technique are compared against the consensus label and show that the techniques are equivalent for the striatum, but the pseudo-MRI based technique yields better results for both the thalamus and the globus pallidus.

The functional validation for both warping techniques yields 31 of 34 (89\%) data points falling inside the atlas definition of the sensory thalamus. The data points warped using inverse atlas-to-template transformation yielded a centroid which was $2.5 \mathrm{~mm}$ away from the center of gravity of the sensory thalamus of the atlas. The points warped back to the atlas space using the inverse pseudo-MRI based transformations yielded a centroid which was $2 \mathrm{~mm}$ away from the center of gravity the sensory thalamus. These results suggest the the pseudo-MRI based method yields a better atlas-to-patient customization.

\section{Conclusions and Future Work}

In this paper we have presented two different techniques to warp an atlas derived from serial histological data to fit clinical patient MRI volumes. The first relies on a template created from the Colin27 MRI average to estimate an atlas-to-patient warp. The second technique uses a pseudo-MRI derived from a voxel-label-atlas and has a contrast similar to the Colin27 MRI average. Both techniques were evaluated using anatomical and electrophysiological data from pre-operative clinical data from Parkinson's patients. The analysis comparing the atlas label to manual labels shows that the pseudo-MRI based warping technique may be better than the template based technique for the striatum, thalamus, and globus pallidus. An analysis of pre-operative electrophysiological data also suggests the 
pseudo-MRI based technique offers increased accuracy over the template based technique.

Additional data will be required to determine if the pseudo-MRI based nonlinear atlas-to-patient warping technique is statistically better than the the Colin27 MRI template based technique, and is the subject of future work. We would like to continue the atlas validation using more intraoperative data, by classifying the electrophysiological data with respect to the strength of the stimulation and the strength of the sensory responses, and to create a probabilistic functional atlas of the basal ganglia and thalamus from this data.

\section{References}

1. J. Atkinson et al. J. of Neurosurg., 96(5):672-680, October 2002.

2. G. Bertrand et al. Trans Am Neurol Assoc, 98:233-237, 1973.

3. F.L. Bookstein. IEEE PAMI, 11(6):567-585, 1989.

4. M.M. Chakravarty et al. In T. Peters and R. Ellis ed., MICCAI LNCS, 2003.

5. M.M. Chakravarty et al. Neuroimage, 2005. Submitted.

6. D.L. Collins and A.C. Evans. IJPRAI, pages 1271-1294, December 1997.

7. S.C.L Deoni et al. MRM, 53:237-241, 2005.

8. E.G. Duerdenet al. In T. Peters and R. Ellis ed., MICCAI LNCS, 2003.

9. K.W. Finnis et al. IEEE TMI, 22(1), January 2003.

10. K.A. Ganser et al. Med Image Anal, 2004.

11. P. Gloor. The Temporal lobe and the limbic system. Oxford University Press, 1997.

12. T. Hirai and E.G. Jones. Brain Res Brain Res Rev., 14(1), 1989.

13. C.J. Holmes et al. J Comput Assist Tomogr, 22(2), 1998.

14. H. Johansen-Berg et al. Cereb Cortex, 15, January 2005.

15. G.L. Nowinski et al. IEEE TMI, 19(1), January 2000.

16. G. Schaltenbrand and W. Wahren. Atlas for Stereotaxy of the Human Brain. Georg Thieme Verlag, 1977.

17. P. St-Jean et al. IEEE TMI, 17, May 1998.

18. J. Talairach and P. Tournoux. Co-Planar Stereotaxic Atlas of the Human Brain. Georg Thieme Verlag, 1988. 